\title{
Three-dimensional flower-like brushite crystals prepared from high internal phase emulsion for drug delivery application
}

\begin{abstract}
In the present study, three-dimensional brushite crystals were prepared through palm olein-inwater high internal phase emulsion processing route for the first time. X-ray diffraction patterns revealed that the powders possessed brushite crystalline phase with trace amount of hydroxyapatite. Unique morphologies of the brushite crystals were obtained as a result of tailoring the precursor concentration, surfactant concentration and oil volume fraction. These factors governed the rate of nucleation and crystal growth, resulting in flower-like morphologies. The petal-like flakes grew radially from the centre which gave rise to porosity of less than $2 \mu \mathrm{m}$. A plausible mechanism of crystal growth is discussed and postulated schematically. Sodium ampicillin, a broad spectrum antibiotic, was loaded into the pores of the crystals, which was subsequently released in vitro. The controlled release ability for up to 14 days indicated the potential of using these brushite crystals as drug delivery agents for localized treatments.
\end{abstract}

Keyword: High internal phase emulsion; Calcium phosphate; Electron microscopy; Porosity; Drug delivery. 